\title{
A CONSTRUCTION OF PSEUDO-ANOSOV HOMEOMORPHISMS
}

\author{
ROBERT C. PENNER
}

\begin{abstract}
We describe a generalization of Thurston's original construction of pseudo-Anosov maps on a surface $F$ of negative Euler characteristic. In fact, we construct whole semigroups of pseudo-Anosov maps by taking appropriate compositions of Dehn twists along certain families of curves; our arguments furthermore apply to give examples of pseudo-Anosov maps on nonorientable surfaces. For each self-map $f: F \rightarrow F$ arising from our recipe, we construct an invariant "bigon track" (a slight generalization of train track) whose incidence matrix is Perron-Frobenius. Standard arguments produce a projective measured foliation invariant by $f$. To finally prove that $f$ is pseudo-Anosov, we directly produce a transverse invariant projective measured foliation using tangential measures on bigon tracks. As a consequence of our argument, we derive a simple criterion for a surface automorphism to be pseudo-Anosov.
\end{abstract}

Introduction. A homeomorphism $\varphi$ of a surface $F$ is said to be pseudo-Anosov if no iterate of $\varphi$ fixes any essential nonboundary- or puncture-parallel free homotopy class of simple curves in $F$. Examples of these homeomorphisms date back to the work of Nielsen (see [ $\mathbf{N}$ and $\mathbf{G i}]$ ), but a systematic study of these maps was not undertaken until the work of Thurston [T1]. Anosov $[\mathbf{A}]$ studied maps of the torus which preserve two foliations of the torus by lines of irrational slope, and pseudo-Anosov maps on $F$ similarly preserve a pair of foliations (with singularities).

Pseudo-Anosov maps are by no means special; indeed, the monodromy of any nontorus fibred knot which is not a satellite is pseudo-Anosov [T4]. (Note that being pseudo-Anosov is a conjugacy invariant.) Moreover, these maps play an important role in the geometrization of three-manifolds; indeed, a mapping torus has hyperbolic structure if and only if the monodromy is pseudo-Anosov [T4].

In the original preprint [T1], there is described a construction of pseudo-Anosov maps which we will recall later. In this paper, we generalize Thurston's construction and give a recipe for constructing whole semigroups of pseudo-Anosov maps, many of which do not arise from Thurston's construction. Our recipe is also applicable to nonorientable surfaces, and we give examples of pseudo-Anosov maps in this setting. (In [T3], Thurston proved the existence of such, and [AY] gave the first explicit examples.)

This paper is organized as follows. In $\S 1$, we review the basic terminology and results on train tracks in surfaces and indicate the connection between measured train tracks and measured foliations. $\S 2$ is devoted to tangential measure on bigon

Received by the editors March 7, 1986 and, in revised form, June 17, 1987.

1980 Mathematics Subject (lassification (1985 Revision). Primary 57N06, 57N50.

Key words and phrases. Pseudo-Anosov, measured foliation, train track, mapping class group, Dehn twist.

The author was partially supported by the National Science Foundation. 
tracks, and we develop a new condition $(\mathrm{AC})$ on tangential measures which is central to later concerns. We then proceed, in $\S 3$, to describe our construction of semigroups of pseudo-Anosov maps for the case of oriented surfaces; as a consequence of the proof, we derive a simple criterion for recognizing pseudo-Anosov maps. We describe an integral linear representation of the semigroups of mapping classes we have constructed and consider the question of whether our recipe gives virtually all pseudo-Anosov maps. Finally, $\S 4$ extends our results to the setting of nonorientable surfaces and gives examples.

This work owes obvious intellectual debts to the far-reaching insights of William Thurston.

1. We begin by recalling the basic definitions and results introduced in [T2 and T3]. See [HP or Pa] for a comprehensive treatment of this material. A train track $\tau$ on a surface $F$ is a finite collection of simple closed curves and one-dimensional CW complexes disjointly embedded in the interior of $F$. The vertices of $\tau$ are called switches, and the (open) edges and the simple closed curve components of $\tau$ are called branches. We furthermore require the following conditions on $\tau$.

(1) (Smoothness) The branches of $\tau$ are $\mathscr{C}^{1}$. If $b_{1}$ and $b_{2}$ are branches of $\tau$ incident on the switch $s$, then the one-sided tangents to $b_{1}$ and $b_{2}$ at $s$ either coincide or differ by rotation-by- $\pi$ in the tangent plane at $s$.

(2) (Nondegeneracy) Each switch of $\tau$ is at least trivalent. For any switch $s$ of $\tau$, there is a $\mathscr{C}^{1}$ embedding $f:(0,1) \rightarrow \tau$ with $f\left(\frac{1}{2}\right)=s$.

(3) (Geometry) If $\mathscr{S}$ is a component of $F-\tau$, then the double of $\mathscr{S}$ along the edges of $\tau$ has negative Euler characteristic.

An example of a train track is given in Figure 3.1b.

An $n$-gon $D$ in $F$ is an open two disc embedded in the interior of $F$ with $n$ discontinuities in the tangent to the frontier of $D$. Condition (3) is equivalent to the condition that no component of $F-\tau$ is a bigon, monogon, nullgon or smooth annulus.

We say a track $\tau$ carries a track $\tau^{\prime}$ if there is a $\mathscr{C}^{1}$ map $\Phi: F \supset$ homotopic to the identity so that $\Phi\left(\tau^{\prime}\right) \subset \tau$ with $d \Phi_{p} \mid$ (tangent to $\left.\tau^{\prime}\right) \neq 0$ for any $p \in \tau^{\prime}$. Suppose $\tau^{\prime}$ is carried by $\tau$ with $\Phi\left(\tau^{\prime}\right) \subset \tau$. We define the incidence matrix $M$ of $\Phi$ relative to $\tau$ and $\tau^{\prime}$ as follows. For each branch $b_{i}$ of $\tau$, choose some point $x_{i}$ in the interior of $b_{i}$. We define $M_{i j}=\#\left\{\Phi^{-1}\left(x_{i}\right) \cap c_{j}\right\}$, where $c_{j}$ is a branch of $\tau^{\prime}$.

If $A$ is a matrix or vector, we will write $A \geq 0$ to mean that each entry of $A$ is nonnegative with a similar interpretation for $A>0$. Thus, $M \geq 0$ by definition. We have the following classical theorem (see $[\mathbf{G a}]$ ), which is a fundamental tool in what follows.

THEOREM A (PERRON-FROBENIUS). If $M \geq 0$ is a square matrix and if there is some positive integer $N$ so that $M^{N}>0$, then the eigenvalue $\lambda$ of $M$ of maximum modulus is positive real. Moreover, the eigenvector $x$ of $M$ corresponding to $\lambda$ satisfies $x>0$, and $x$ is the unique eigenvector with this property.

A train track $\tau$ is recurrent if for each branch $b$ of $\tau$, there is some curve $\gamma$ carried by $\tau$ with $b \subset \Phi(\gamma)$. If $\tau^{\prime} \subset F$ is another train track which is transverse to $\tau$, we say $\tau$ hits $\tau^{\prime}$ efficiently if there is no bigon in $F$ with frontier made of two $\mathscr{C}^{1}$ arcs, one from $\tau^{\prime}$ and one from $\tau$. A train track $\tau$ is transversely recurrent if for each branch $b$ of $\tau$ there is a simple curve $\gamma$ so that $\gamma \cap b \neq \varnothing$ and $\gamma$ hits $\tau$ efficiently. Transverse 


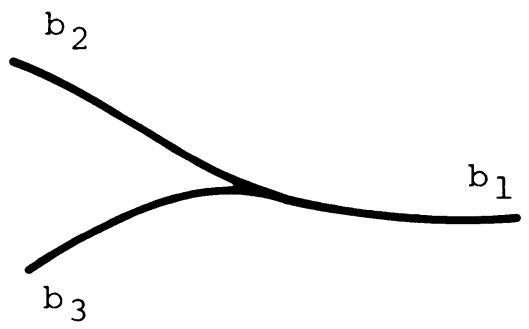

FIGURE 1.1

recurrence will be discussed in $\S 2$. A recurrent and transversely recurrent track will be called birecurrent. A track $\tau$ is said to fill $F$ if each component of $F-\tau$ is a disc, a boundary-parallel annulus, or a puncture-parallel punctured disc.

A transverse measure (or simply a measure) $\mu$ on a track $\tau$ is a function

$$
\mu:\{\text { branches of } \tau\} \rightarrow \mathbf{R}^{+} \cup\{0\}
$$

satisfying a certain equality (called a switch condition) at each switch; namely, if branches $b_{1}, b_{2}, b_{3}$ of $\tau$ are as indicated in Figure 1.1, then $\mu\left(b_{1}\right)=\mu\left(b_{2}\right)+\mu\left(b_{3}\right)$. It is easy to see that a train track is recurrent if and only if it supports a positive measure.

A measure $\mu>0$ on a track $\tau$ defines a measured foliation in $F$ as follows. (See [FLP] for the theory of measured foliations.)

Construction. Choose a metric on $F$ and consider the rectangular neighborhood $N_{i}$ of branch $b_{i}$ of $\tau$ of width $\mu\left(b_{i}\right)$ foliated by arcs parallel to $b_{i}$. We may assume (by rescaling the metric if necessary) that the $N_{i}$ are pairwise disjoint except near the switches. The switch conditions guarantee that the $N_{i}$ may be combined to give a foliated neighborhood $(N, \mathscr{F})$ of $\tau$ as in Figure 1.2a. We identify the edges of complementary regions of $F-N$ as in Figure $1.2 \mathrm{~b}$ to get a measured foliation of $F$.

Every (equivalence class of a) measured foliation on $F$ arises in this way from a measure on some birecurrent track. Moreover, distinct measures on a fixed track define distinct classes of measured foliations. In contrast, distinct train tracks can support measures which describe the same class. We define an equivalence relation

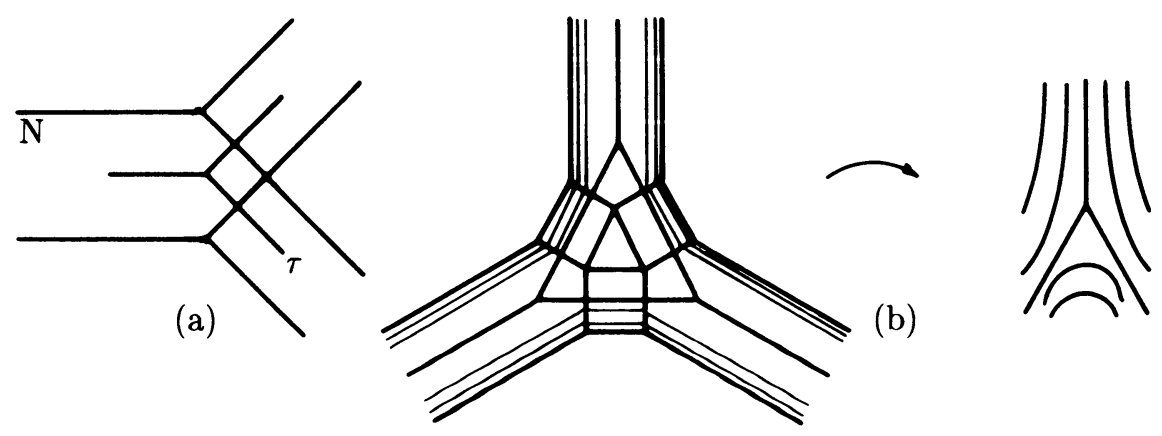

FIGURE 1.2 

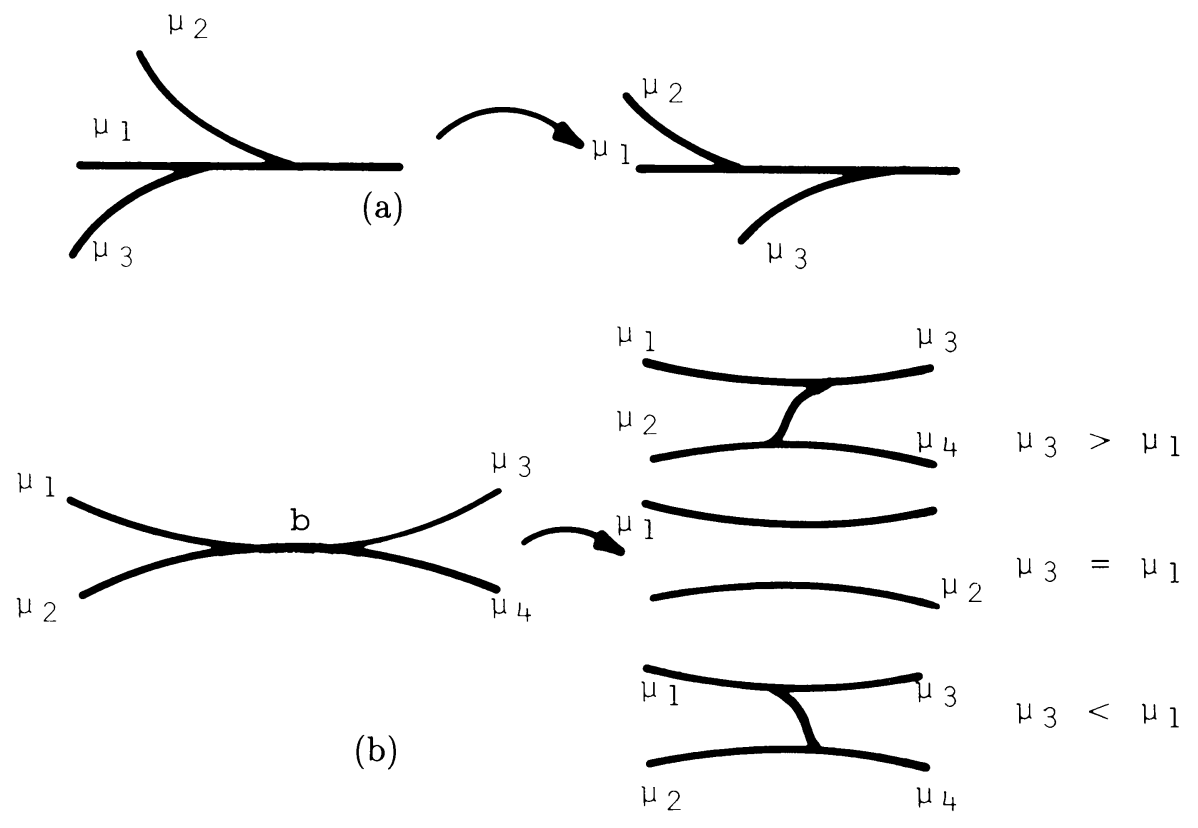

FIGURE 1.3

$\sim$ on measured tracks generated by three moves: isotopy, splitting, and shifting, to be defined presently. It will be clear that equivalent tracks define equivalent measured foliations on $F$ (and the converse is proved in [HP]).

To shift a measured track, we slide one switch past another as in Figure 1.3a. (Numbers next to edges indicate measures.) There are three cases of splitting a branch $b$ as in Figure 1.3b: $\mu_{3}>\mu_{1}$ (and $\mu_{2}>\mu_{4}$ by the switch condition); $\mu_{3}=\mu_{1}\left(\mu_{2}=\mu_{4}\right)$; and $\mu_{3}<\mu_{1}\left(\mu_{2}<\mu_{4}\right)$. We split as indicated in the figure in these cases. The inverse of a split is called a collapse, and the collapse is called nontrivial if it is not of the second type.

REMARK. If $(\tau, \mu)$ splits to $\left(\tau^{\prime}, \mu^{\prime}\right)$, then $\tau$ carries $\tau^{\prime}$.

One constructs the space $\mathscr{M T}_{0}(F)$ of measured tracks as the space of equivalence classes; denote the class of $(\tau, \mu)$ by $[\tau, \mu] . \mathscr{M T}_{0}(F)$ inherits a topology from the Euclidean topology of measures on tracks. There is an associated projective space $\mathscr{P} \mathscr{T}_{0}(F)=\mathscr{M} \mathscr{T}_{0}(F)-\{0\} / \mathbf{R}^{+}$of projective measured tracks, where $\mathbf{R}^{+}$acts multiplicatively on measures. $\mathscr{P}_{0}(F)$ is a sphere which compactifies the Teichmüller space of $F$. (See $[\mathbf{T 1}, \mathbf{F L P}, \mathbf{K}]$.) The action of homeomorphisms of $F$ on $\mathscr{M} \mathscr{T}_{0}(F)$ descends to an action of the mapping class group $M C(F)$ on $\mathscr{M G}_{0}(F)$ or $\mathscr{P} \mathscr{T}_{0}(F)$, which continuously extends the usual action of $M C(F)$ on the Teichmüller space. (See $[\mathbf{K}]$.)

The space $M \mathscr{T}_{0}(F)\left(P \mathscr{T}_{0}(F)\right)$ is canonically isomorphic to the space $M \mathscr{F}_{0}(F)$ $\left(P \mathscr{F}_{0}(F)\right)$ of measured (projective) foliations of compact support on $F$ considered in [FLP]. The collection of measures supported on a birecurrent maximal (in the sense of inclusion of point sets) track is often regarded as a coordinate patch on the manifold $M \mathscr{F}_{0}(F)$. 
There is a natural, bicontinuous and symmetric pairing

$$
\langle\cdot, \cdot\rangle: \mathscr{M S}_{0}(F) \times \mathscr{M} \mathscr{T}_{0}(F) \rightarrow \mathbf{R}^{+} \cup\{0\}
$$

which extends the geometric intersection number of curves. In case $\mu$ and $\mu^{\prime}$ are measures on tracks $\tau$ and $\tau^{\prime}$ hitting efficiently, then

$$
\left\langle[\tau, \mu],\left[\tau^{\prime}, \mu^{\prime}\right]\right\rangle=\sum_{\substack{\text { branches } b_{j} \text { of } \tau \\ \text { branches } b_{i}^{\prime} \text { of } \tau^{\prime}}} \#\left(b_{i}^{\prime} \cap b_{j}\right) \mu^{\prime}\left(b_{i}^{\prime}\right) \mu\left(b_{j}\right) .
$$

A mapping class $\Psi$ on a surface $F$ of negative Euler characteristic is said to be pseudo-Anosov (abbreviated pA) if there is a pair $\mathscr{F}_{s}$ and $\mathscr{F}_{u}$ of transverse arational (i.e., no closed leaves) measured foliations on $F$ and a representative $f$ of $\Psi$ so that $f\left(\mathscr{F}_{u}\right)=\lambda \mathscr{F}_{u}$ and $f\left(\mathscr{F}_{s}\right)=\mathscr{F}_{s} / \lambda$ with $\lambda>1 . \quad \lambda$ is called the dilatation of $\Psi$. (See [Mi and HT] for a proof that this definition agrees with the definition in the introduction.) A mapping class $\Psi$ is reducible if there is a family of essential disjoint simple closed nonboundary- or puncture-parallel curves $C$ and a representative $f$ of $\Psi$ with $f(C)=C$. In case there is such a collection $C$ of reducing curves and $S$ is a component of $F-C$, we may choose some least iterate $f^{N}$ of $f$ that maps $S$ to $S$ fixing $C \cap S$ componentwise. The isotopy class of $\left.F^{N}\right|_{S}$ is called a component map of $\Psi$. Thurston's classification, (see [Gi or FLP]), of mapping classes says that each component map of $\Psi$ is either periodic or pA.

2. We will call a branched one-submanifold $\tau$ of $F$ that may fail to be a train track only because of bigon complementary regions a bigon track (or simply a track). All the notions and constructions of the previous section apply to bigon tracks. Unlike train tracks, however, distinct measures on a bigon track can describe equivalent measured foliations. Similarly, when we say that one bigon track carries another, we must be careful to specify how the track is carried.

A tangential measure $\nu$ on a bigon track $\tau$ is a function

$$
\nu:\{\text { branches of } \tau\} \rightarrow \mathbf{R}^{+} \cup\{0\}
$$

satisfying the following conditions:

(i) Let $D$ be an $n$-gon, $n>1$, of $F-\tau$ with $\mathscr{C}^{1}$ frontier edges $E_{1}, \ldots, E_{n} \subset \tau$, where $E_{i}$ is the closure of the union of branches $b_{i, j}, j=1, \ldots, r_{i}$ of $\tau$. Define the total tangential measure of $E_{i}$ by $\nu\left(E_{i}\right)=\sum_{j} \nu\left(b_{i, j}\right)$. We require that $\nu\left(E_{i}\right) \leq$ $\sum_{k \neq i} \nu\left(E_{k}\right)$, for each $i$.

(ii) Let $A$ be a topological annulus of $F-\tau$ with frontier the union of $\operatorname{arcs} \gamma_{1}$ and $\gamma_{2}$ so that $\gamma_{1}$ is smooth. We require $\nu\left(\gamma_{1}\right) \leq \nu\left(\gamma_{2}\right)$.

Just as with measure and recurrence, a track is transversely recurrent if and only if it supports a positive tangential measure satisfying strict inequalities. (See [HP].)

If $\tau$ is a bigon track which fills $F$, we define the dual bigon track $\tau^{*}$ as follows. Start with a short transversal $b_{i}^{*}$ to each branch $b_{i}$ of $\tau$ so that $b_{i}^{*} \cap b_{j}=\delta_{i, j}$. Let $R$ be a simply connected region of $F-\tau$ and let $E$ be an edge of $R$, where $E$ is the closure of the branches $b_{1}, \ldots, b_{n}$. The transversals $b_{i}^{*}, i=1, \ldots, n$, are all made confluent in $R$ near $\tau$ to form a branch, say $e$, of $\tau^{*}$. Finally, if $e_{1}$ and $e_{2}$ correspond to adjacent edges $E_{1}$ and $E_{2}$ of $R$, then we add a branch to $\tau^{*}$ smoothly connecting $e_{1}$ and $e_{2}$ in $R$. A similar procedure is employed if $R$ is an annulus. 


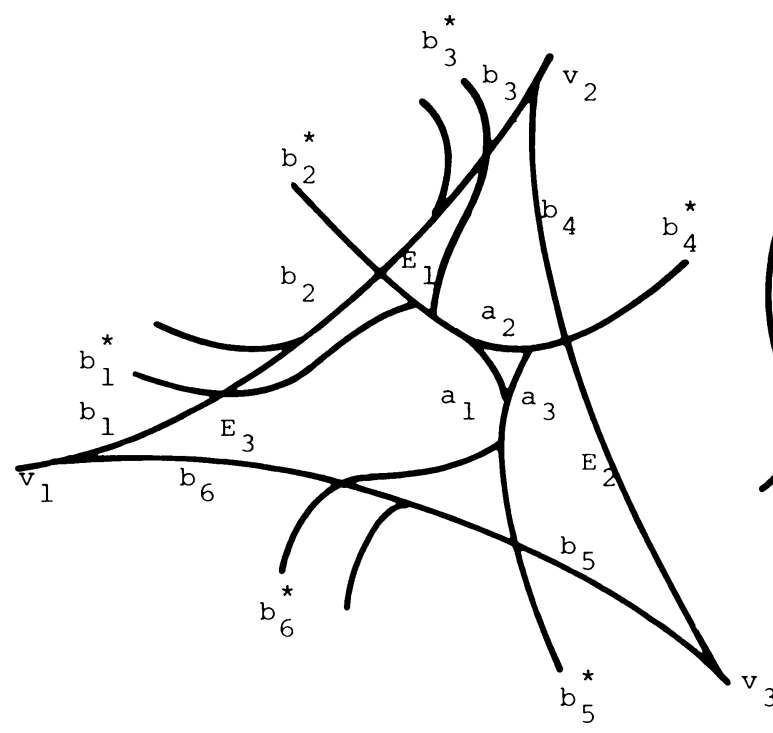

(a)

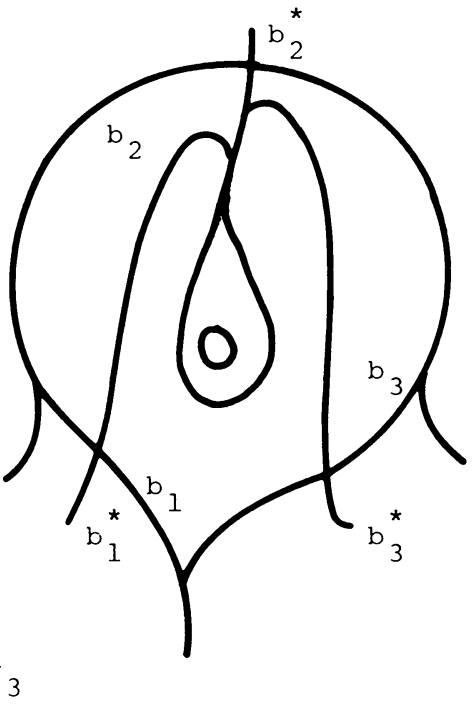

(b)

FIGURE 2.1

Some examples are given in Figure 2.1. Notice that $\tau^{*}$ has one complementary bigon for each switch of $\tau$, and $\tau^{*}$ hits $\tau$ efficiently.

Certain tangential measures $\nu$ on $\tau$ determine (transverse) measures $\nu^{*}$ on $\tau^{*}$ so that

$$
\left\langle[\tau, \mu],\left[\tau^{*}, \nu^{*}\right]\right\rangle=\sum_{\substack{\text { branches } \\ b_{i} \text { of } \tau}} \mu\left(b_{i}\right) \nu\left(b_{i}\right)
$$

for any measure $\mu$ on $\tau$. The salient condition is the following:

Alternating Condition AC. Suppose $R$ is a simply connected component of $F-\tau$ and the consecutive edges of the frontier of $R$ have total tangential measure $\left(\nu_{i}\right)_{i=1}^{n}$; regard the subscripts as cyclically ordered. We require

(a) $\sum_{i=j}^{n+j-1}(-1)^{i-j} \nu_{i} \geq 0, j=1, \ldots, n$,

(b) $\nu_{j} \leq \nu_{j-1}+\nu_{j+1}, j=1, \ldots, n$.

In particular, if $n$ is even, then the sum in (a) is zero. $\mathrm{AC}$ is equivalent to the triangle inequalities on a 3-gon and on a 4-gon equivalent to $\nu_{1}+\nu_{3}=\nu_{2}+\nu_{4}$. A tangential measure on $\tau$ satisfying AC is called a metric on $\tau$.

LEMMA 2.1. Let $\tau$ be a bigon track filling $F$ and let $\nu$ be a metric on $\tau$. $\nu$ determines a collection $\mathscr{M}(\nu)$ of measures on $\tau^{*}$ so that for each $\nu^{*} \in \mathscr{M}(\nu)$ $\nu^{*}\left(b^{*}\right)=\nu(b)$, if $b$ is a branch of $\tau$. Moreover, $\mathscr{M}(\nu)$ is a singleton if $\tau$ has only complementary oddgons, and there is a one-parameter family of solutions in $\mathscr{M}(\nu)$ for each evengon.

PROOF. Suppose $R$ is an $n$-gon of $F-\tau$, and adopt the notation of Figure 2.2a for the branches of $\tau^{*}$. Solving for $\left(\nu^{*}\left(a_{i}\right)\right)_{1}^{n}$ in terms of $\left(\nu_{i}=\nu\left(E_{i}\right)\right)_{1}^{n}$ yields the 
linear system

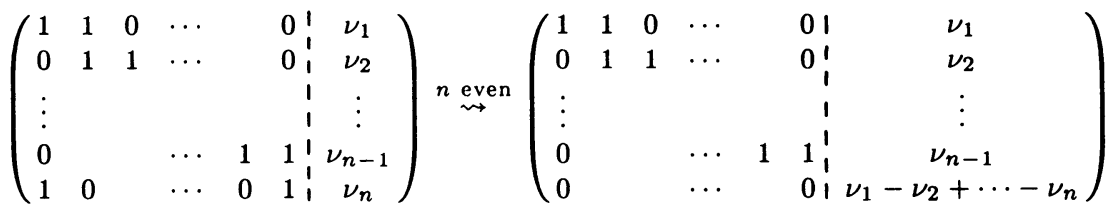

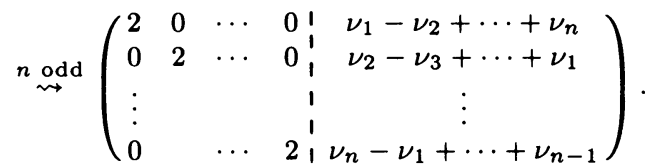

Thus, AC guarantees a unique (and nonnegative) solution on an oddgon.

On an evengon, we find a one-dimensional solution space to the linear system, and we must verify the existence of a nonnegative solution. We proceed by induction on the number of sides of the $2 n$-gon, and the case of a bigon is an easy exercise. Consider a fourgon of consecutive sides $E_{1}, \ldots, E_{4}$ as in Figure 2.2a. We see that

$$
\begin{aligned}
& \nu^{*}\left(a_{1}\right)=t, \quad \nu^{*}\left(a_{2}\right)=\nu\left(E_{1}\right)-t, \\
& \nu^{*}\left(a_{3}\right)=\nu\left(E_{2}\right)-\nu\left(E_{1}\right)+t, \\
& \nu^{*}\left(a_{4}\right)=\nu\left(E_{3}\right)-\nu\left(E_{2}\right)+\nu\left(E_{1}\right)-t
\end{aligned}
$$

is a solution which is nonnegative provided

$$
\sup \left\{0,\left(\nu\left(E_{1}\right)-\nu\left(E_{2}\right)\right)\right\} \leq t \leq \inf \left\{\nu\left(E_{4}\right), \nu\left(E_{1}\right)\right\},
$$

and the claim follows on a 4-gon.

If $R$ is a $2 n$-gon of more than four sides, let $f$ be an edge of $R$ with $\nu(f)$ largest. The diagonal $d$ opposite $f$ decomposes $R$ into a 4-gon $Q$ and $(2 n-2)$-gon $P$, as in Figure 2.2b. Defining $\nu(d)=\nu(e)-\nu(f)+\nu(g)$, we see that $\nu$ satisfies AC on both $P$ and $Q$, and the inductive hypothesis yields a measure $\mu^{\prime}$ on $\tau^{\prime} \cap P$, where $\tau^{\prime}$ is the track indicated in Figure 2.2b. Since $\nu(f)$ is largest, a short computation shows that we can extend $\mu^{\prime}$ on $\tau^{\prime} \cap P$ to $\mu^{\prime \prime}$ on $\tau^{\prime}$ so that $\mu^{\prime \prime}(\beta)=\mu^{\prime \prime}(\alpha)$, where $\alpha$ and $\beta$ are the branches of $\tau^{\prime}$ indicated. Splitting along the branch of $\tau^{\prime}$ which connects $\alpha$ and $\beta$ proves the claim.

Finally, in case $R$ is a boundary- or puncture-parallel region of $F-\tau$, it is an exercise to find a unique $\nu^{*}$ on $\tau^{*} \cap R$ with $\nu^{*}\left(b_{i}^{*}\right)=\nu\left(b_{i}\right)$, proving the lemma.

Suppose $\psi$ is a homeomorphism of $F$ and $\psi(\tau)$ collapses, by nontrivial collapses, to $\tau$ for some track $\tau$ in $F$ with branches $\left(b_{i}\right)_{1}^{n}$. The $n \times n$ incidence matrix $M$ of the collapse of $\psi(\tau)$ onto $\tau$ describes the natural action of $\psi$ on measures on $\tau$ in the sense that $\psi([\tau, \mu]) \sim[\tau, M \mu]$, where $\mu=\left(\mu\left(b_{i}\right)\right)_{1}^{n}$. We have also

LEMMA 2.2. $M^{t}$ describes the natural action of $\psi^{-1}$ on metrics in the following sense: if $\nu$ is a metric on $\tau$ and $\nu^{*} \in \mathscr{M}(\nu)$, then $\psi^{-1}\left(\left[\tau^{*}, \nu^{*}\right]\right) \sim\left[\tau^{*}, \bar{\nu}\right]$, where $\bar{\nu} \in \mathscr{M}\left(M^{t} \nu\right)$.

Proof. Suppose $\tau^{\prime}=\psi(\tau)$ differs from $\tau$ by a single collapse and label the salient branches of $\tau$ and $\tau^{\prime}$ as in Figure 2.3. If $\mu^{\prime}$ is a measure on $\tau^{\prime}$ and $\mu$ the 


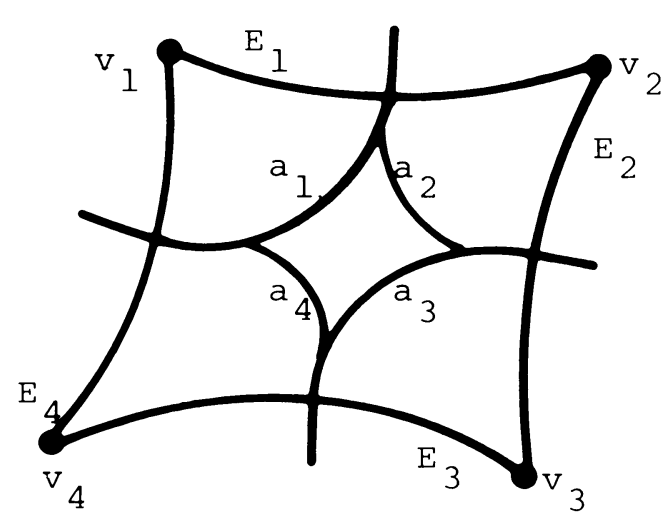

(a)

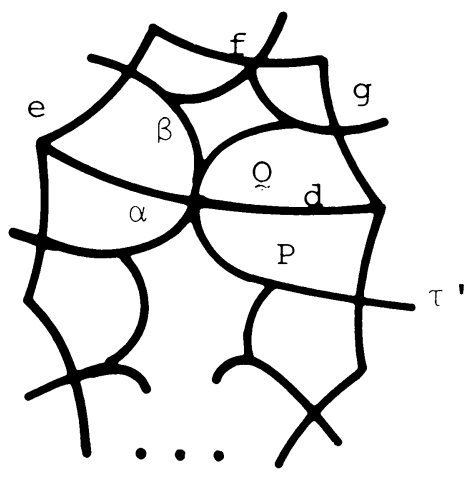

(b)

FIGURE 2.2
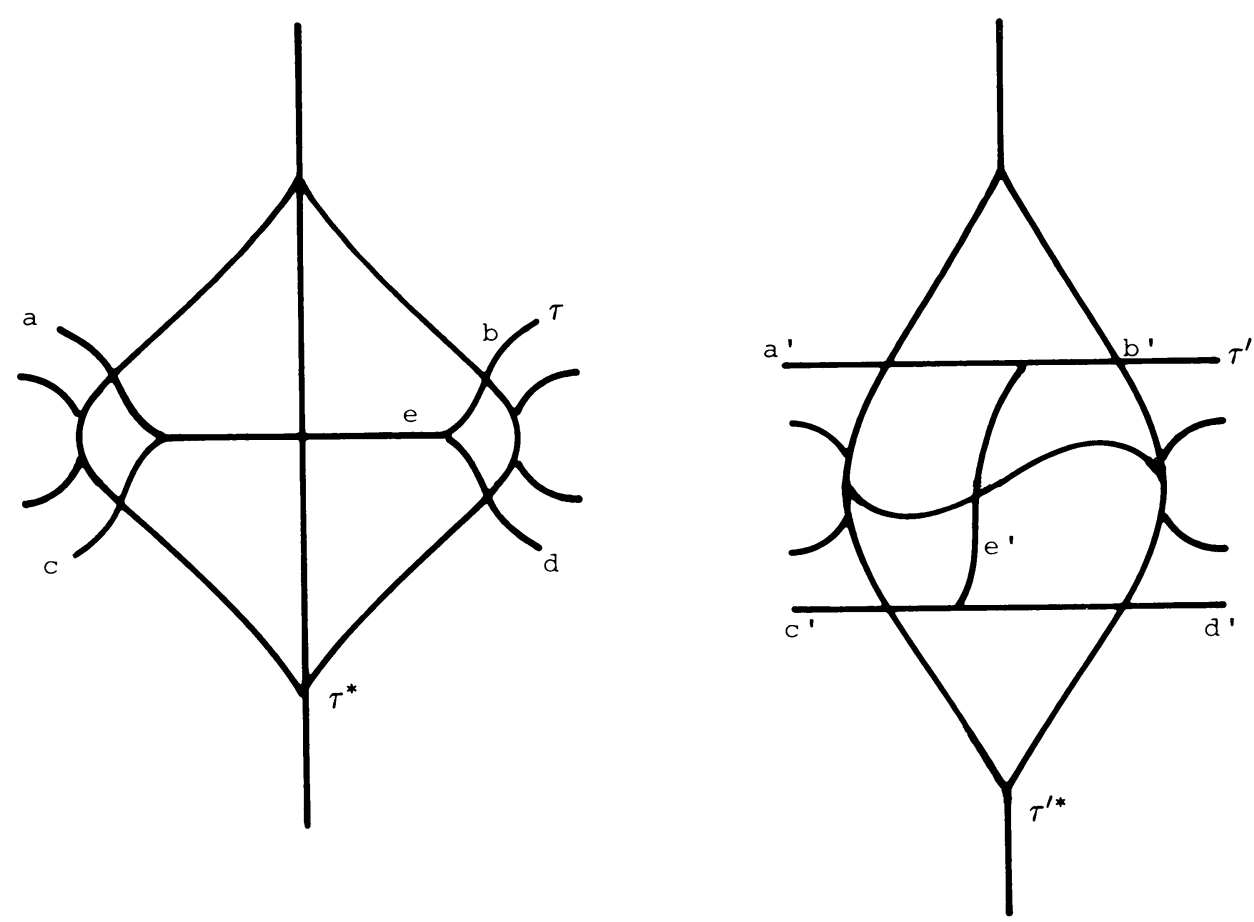

FigURE 2.3 
corresponding measure on $\tau$, then

$$
\left(\begin{array}{l}
\mu(a) \\
\mu(b) \\
\mu(c) \\
\mu(d) \\
\mu(e)
\end{array}\right)=\left(\begin{array}{lllll}
1 & 0 & 0 & 0 & 0 \\
0 & 1 & 0 & 0 & 0 \\
0 & 0 & 1 & 0 & 0 \\
0 & 0 & 0 & 1 & 0 \\
1 & 0 & 0 & 1 & 1
\end{array}\right)=\left(\begin{array}{c}
\mu^{\prime}\left(a^{\prime}\right) \\
\mu^{\prime}\left(b^{\prime}\right) \\
\mu^{\prime}\left(c^{\prime}\right) \\
\mu^{\prime}\left(d^{\prime}\right) \\
\mu^{\prime}\left(e^{\prime}\right)
\end{array}\right) .
$$

Consider now the tracks $\tau^{*}$ and $\tau^{\prime *}$ indicated in Figure 2.3. We see that $\left[\tau^{*}, \nu^{*}\right] \sim$ $\left[\tau^{\prime *}, \bar{\nu}\right]$ with $\bar{\nu} \in \mathscr{M}\left(\nu^{\prime}\right)$, where

$$
\left(\begin{array}{l}
\nu^{\prime}\left(a^{\prime}\right) \\
\nu^{\prime}\left(b^{\prime}\right) \\
\nu^{\prime}\left(c^{\prime}\right) \\
\nu^{\prime}\left(d^{\prime}\right) \\
\nu^{\prime}\left(e^{\prime}\right)
\end{array}\right)=\left(\begin{array}{lllll}
1 & 0 & 0 & 0 & 1 \\
0 & 1 & 0 & 0 & 0 \\
0 & 0 & 1 & 0 & 0 \\
0 & 0 & 0 & 1 & 1 \\
0 & 0 & 0 & 0 & 1
\end{array}\right)\left(\begin{array}{c}
\nu(a) \\
\nu(b) \\
\nu(c) \\
\nu(d) \\
\nu(e)
\end{array}\right),
$$

and the lemma follows once we check that $M^{t} \nu$ is a metric on $\tau^{\prime}$. Since the effect of the collapse on total tangential measures of edges of complementary regions of $F-\tau^{\prime}$ is to add a constant $\nu(e)$ to those of two adjacent edges of $F-\tau$, the three conditions for tangential measure and the two conditions for $\mathrm{AC}$ are invariant; the lemma follows.

3. If $c$ is an embedded curve in an oriented surface $F$, then we recall that the "Dehn twist" $\tau_{c}$ on $c$ is the mapping class on $F$ corresponding to cutting $F$ along $c$, twisting to the right by $2 \pi$, and regluing. The main result of this paper follows.

THEOREM 3.1. Suppose that $\mathscr{C}$ and $\mathscr{D}$ are each disjointly embedded collections of essential simple closed curves (with no parallel components) in an oriented surface $F$ so that $\mathscr{C}$ hits $\mathscr{D}$ efficiently and $\mathscr{C} \cup \mathscr{D}$ fills $F$. Let $R\left(\mathscr{C}^{+}, \mathscr{D}^{-}\right)$be the free semigroup generated by the Dehn twists $\left\{\tau_{c}^{+1}: c \in \mathscr{C}\right\} \cup\left\{\tau_{d}^{-1}: d \in \mathscr{D}\right\}$. Each component map of the isotopy class of $w \in R\left(\mathscr{C}^{+}, \mathscr{D}^{-}\right)$is either the identity or $p A$, and the isotopy class of $w$ is itself $p A$ if each $\tau_{c}^{+1}$ and $\tau_{d}^{-1}$ occur at least once in $w$.

REMARK. In Theorem 3.4 below, we will describe the kernel of the natural projection from $R\left(\mathscr{C}^{+}, \mathscr{D}^{-}\right)$to the mapping class group.

EXAMPLE. Let $\mathscr{C}$ and $\mathscr{D}$ be the collections of curves illustrated in Figure 3.1a. The word $w=\tau_{c_{1}}^{+2} \tau_{d_{1}}^{-2} \tau_{c_{2}}^{+1} \tau_{c_{3}}^{+1} \tau_{d_{3}}^{-2} \tau_{d_{2}}^{-2} \tau_{c_{2}}^{+3}$ describes a $\mathrm{pA}$ mapping class, for instance. A train track $\tau$ so that $w(\tau)$ is carried by $\tau$ is given in Figure 3.1b.

PROOF. We make two simplifying assumptions. We assume that no component of $\mathscr{C}$ or $\mathscr{D}$ is boundary-parallel or puncture-parallel in $F$. (Twisting on such a component neither affects whether $w$ is $\mathrm{pA}$ nor affects the stable or unstable foliations of $w$ if it is $\mathrm{pA}$.) We also assume that the curves along which we twist in the composition $w$ fill $F$. (If not, we let $F^{\prime} \subset F$ be a regular neighborhood of these curves in $F$. The essential components of $\partial F^{\prime} \subset F$ are then reducing curves, and the argument we give below shows that $\left.w\right|_{F^{\prime}}$ is $\mathrm{pA}$ on components of $F^{\prime}$ that are not annuli.)

We begin by describing the construction of a branched one-submanifold $T$ of $F$. Isotope $C \cup D$ to general position so that $C$ and $D$ still hit efficiently, and consider a point $p=c \cap d$ of $C \cap D$. We introduce branching in $T$ as indicated in Figure 


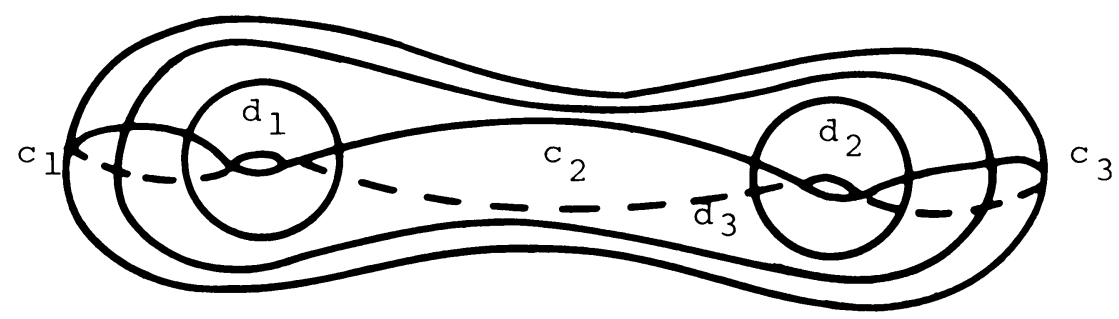

(a)

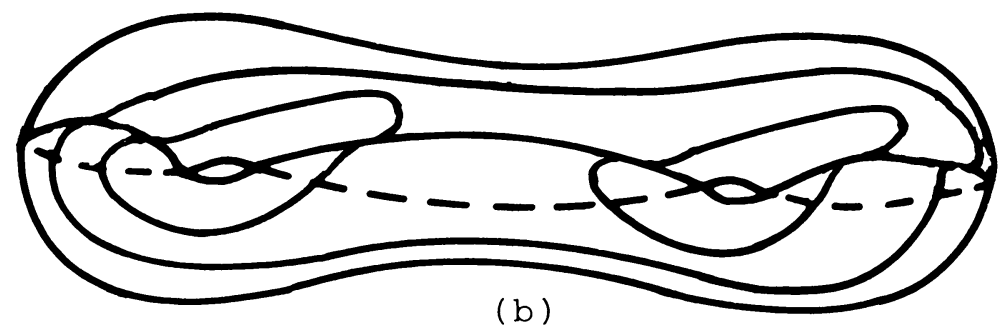

FIGURE 3.1

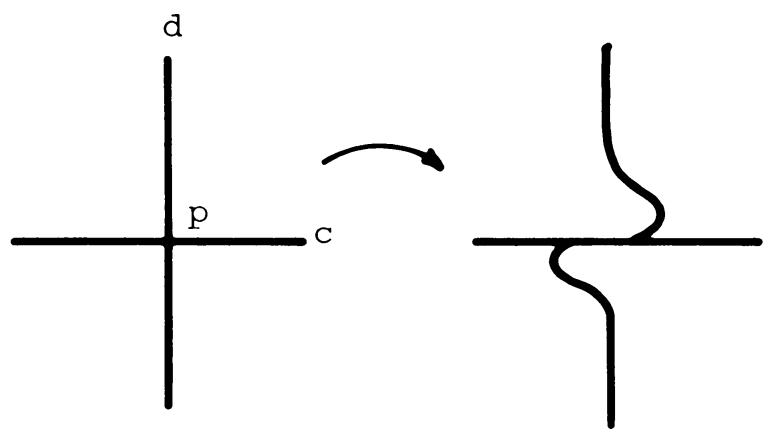

FIGURE 3.2

3.2 so that an arc in $T$ goes smoothly to the right as it approaches $c$ and smoothly to the left as it approaches $d$. The configuration shown in Figure 3.3 gives rise to a bigon complementary region to $T$, and a simple combinatorial argument shows that this is the only type of bad complementary region. Thus, $T$ is a bigon track, which is readily seen to be birecurrent.

We next consider the action of $w$ on the bigon track $T$. Suppose that $\tau_{c}^{+1}$ occurs in the composition $w$, and consider a neighborhood $N(c)$ of $c$ in $F$. In Figure $3.4 \mathrm{a}$, we illustrate a typical case of $T \cap N(c)$, and in Figure $3.4 \mathrm{~b}$, we illustrate 


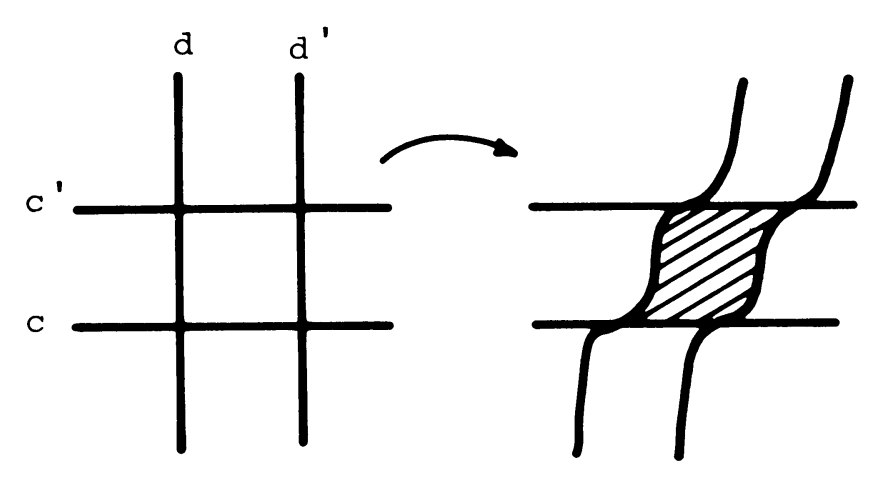

FIGURE 3.3

$\tau_{c}^{+1}(T) \cap N(c) \cdot \tau_{c}^{+1}(T)$ is carried by $T$, and by transitivity of carrying, it follows that $w(T)$ is itself carried by $T$.

Since the pA property is invariant under iteration of mapping classes, it is sufficient to consider a special representative $w^{2}$ of the square of the isotopy class $\left[w^{2}\right]$, and $w^{2}$ is described as follows. A neighborhood $N(c)$ of a curve $c \in \mathscr{C} \cup \mathscr{D}$ is depicted in Figure 3.4a, and the components of $N(c)$ give rise to a pair $c_{ \pm}$of representatives of $[c]$. To define $w^{2}$, we must simply guarantee that for each $\tau_{c}^{ \pm 1}$ that occurs in $w$, both of $\tau_{c_{ \pm}}^{ \pm 1}$ occur in $w^{2}$.

Our convention for carrying $w^{2}(T)$ to $T$ by $\Phi$ is as follows: if $b$ is a branch of $\tau_{c_{ \pm}}^{ \pm 1}(T)$ incident on $c \in \mathscr{C} \cup \mathscr{D}$, then $(T \cap \Phi(b)) \subset(c \cup b)$. Thus, for any measure $\mu$ on $T, w^{2}(T, \mu)$ collapses (by nontrivial collapses) and isotopes to $\left(T, \mu^{\prime}\right)$ for some measure $\mu^{\prime}$. We order the branches $\left\{b_{i}\right\}_{1}^{n}$ of $T$ once and for all and regard a measure $\mu$ on $T$ as a vector $\left(\mu\left(b_{1}\right), \ldots, \mu\left(b_{n}\right)\right)$. With these identifications, the assignment $\mu \mapsto \mu^{\prime}$ is a linear map given by the incidence matrix $M \geq 0$. Note that $M$ depends on our choice of convention of how $T$ carries $w^{2}(T)$.

We claim that $M^{J}>0$ for some $J>0$. Since we twist on each $\tau_{c_{ \pm}}^{ \pm 1}$ for each $\tau_{c}^{ \pm 1} \in w, c \in \mathscr{C} \cup \mathscr{D}$, if $M^{J} \ngtr 0$ for some $J>0$, then $M^{J+1}$ has at least one more nonzero entry than $M^{J}$, and our claim follows. By the Perron-Frobenius Theorem, the eigenvalue $\lambda$ of $M$ of maximum modulus is positive real, and the corresponding (unit) eigenvector $\mathbf{x}=\left(x_{1}, \ldots, x_{n}\right)$ is the unique eigenvector of $M$ with the property $\mathbf{x}>0$. Note that $\lambda>1$ since $M$ is integral and $n>1$.

We define a function $\mu:\left\{b_{i}\right\}_{1}^{n} \rightarrow \mathbf{R}^{+} \cup\{0\}$ by $\mu\left(b_{i}\right)=x_{i}$ and claim that $\mu$ defines a measure on $T$. To each $c \in \mathscr{C}$ there is a unique curve $t(c) \subset T$ isotopic to $c$ with $t(c)-N(\mathscr{C} \cup \mathscr{D}) \subset \mathscr{C}$ (and similarly for $d \in \mathscr{D}$ ). Choose some $c \in \mathscr{C}$, and consider the measure $\mu_{0}$ on $T$ defined by

$$
\mu_{0}\left(b_{i}\right)= \begin{cases}1, & b_{i} \subset t(c), \\ 0, & \text { else }\end{cases}
$$

for $i=1, \ldots, n$. Consider the sequence $\mu^{m}, m \geq 1$, of measures on $T$ which arise from collapsing $w^{m}\left(T, \mu_{0}\right)$ onto $\left(T, \mu^{m}\right)$ using our conventions. Thus $\mu^{m}=M^{m} \mu_{0}$, so $\mu^{m}$ converges projectively to $\mathbf{x}$. Satisfying the switch conditions is a projectively invariant closed condition, and our claim follows. 


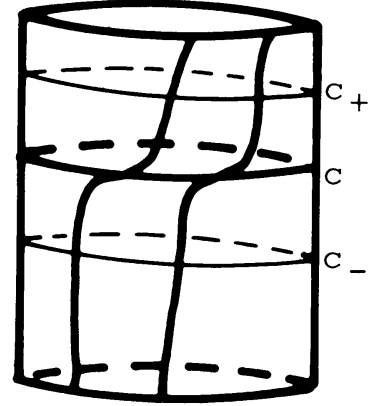

(a)

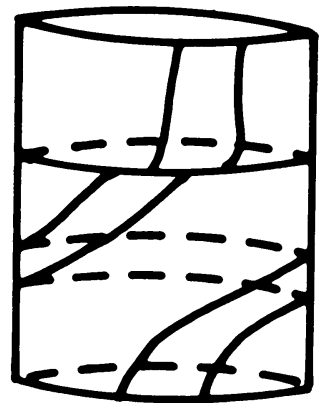

(b)

FIGURE 3.4

Analogously, identify a metric $\nu$ on $T$ with the tuple $\left(\nu\left(b_{i}\right)\right)_{1}^{n}$ and consider the action of $w^{-1}$ on metrics on $T$. Lemma 2.2 assures us that the action is described by $M^{t}$. As before, $M^{t}$ is a Perron-Frobenius matrix, and the positive eigenvector $\mathbf{y}=\left(y_{i}\right)_{1}^{n}$ again has eigenvalue $\lambda$. We claim that $\nu\left(b_{i}\right)=y_{i}, i=1, \ldots, n$, is a metric. To construct a metric $\nu_{0}$ on $T$, we choose a curve $c \in \mathscr{C}$ (or perhaps $d \in \mathscr{D}$ ) so that $\#(c \cap \mathscr{D})>1$ (or perhaps $\#(d \cap \mathscr{C})>1$ ). If there is no such $c \in \mathscr{C} \cup \mathscr{D}$, then $F$ is either the torus or punctured torus, and one readily constructs a metric on $T$. More generally, if $c \in \mathscr{C}$ is as above, then we define $\nu_{0}(b)=\#\left(b \cap c_{+}\right)$. It is easy to verify the metric conditions for $\nu_{0}$. As before, since $\left(M^{t}\right)^{m} \nu_{0} \rightarrow \mathbf{y}$ as $m \rightarrow \infty$, $\mathrm{AC}$ is preserved under splitting (by Lemma 2.2), and AC is a closed condition; our claim follows.

Now, recall the foliated neighborhood $(N, \mathscr{F})$ of a measured track constructed in $\S 1$. By choosing the neighborhood of $T$ more carefully, we construct a bifoliated neighborhood $\left(N, \mathscr{F}, \mathscr{F}{ }^{\perp}\right)$ of $T$ in $F$ so that the rectangle $N_{i}$ about branch $b_{i}$ has width $\mu\left(b_{i}\right)$ (as in $\S 1$ ) and length $\nu\left(b_{i}\right)$. We wish to construct a continuous map $f: F \supset$ mapping $N$ onto itself which is homotopic to $w$ so that $f(\mathscr{F})=\lambda \mathscr{F}$ and $f\left(\mathscr{F}^{\perp}\right)=\lambda^{-1} \mathscr{F} \perp . f$ will not be one-to-one, and the locus in $N$ over which $f$ is not monic will consist of arcs contained in the singular leaves of $\mathscr{F}$. We begin by defining $f$ on $N$ and first require some generalities.

Consider a bigon track $S^{\prime}$ with bifoliated neighborhood arising as above from the measure $\mu^{\prime}$ and metric $\nu^{\prime}$ on $S^{\prime}$. Suppose $e^{\prime}$ is a branch of $S^{\prime}$ which we would like to collapse with incident branches $a^{\prime}, b^{\prime}, c^{\prime}, d^{\prime}$ as indicated in Figure 3.5a. Suppose that $\nu^{\prime}\left(e^{\prime}\right)<\inf \left\{\nu^{\prime}\left(a^{\prime}\right), \nu^{\prime}\left(b^{\prime}\right), \nu^{\prime}\left(c^{\prime}\right), \nu^{\prime}\left(d^{\prime}\right)\right\}$, and consider the arcs $\alpha, \beta, \gamma, \delta$ of $\nu^{\prime}$-length $\nu^{\prime}\left(e^{\prime}\right)$ which are indicated in Figure 3.5a. Identify $\alpha$ to $\beta$ and $\gamma$ to $\delta$ isometrically for $\nu^{\prime}$-length to obtain a bifoliated neighborhood of a bigon track $S$ as indicated in Figure 3.5b. The track $S$ arises from the collapse of $S^{\prime}$ along $e^{\prime}$, and the measure and metric on $S$ corresponding to the bifoliated neighborhood arise from the linear maps derived in Lemma 2.2. We say that the bifoliated neighborhood about $S$ arises from the bifoliated neighborhood about $S^{\prime}$ by zipping along the branch $e^{\prime}$. 


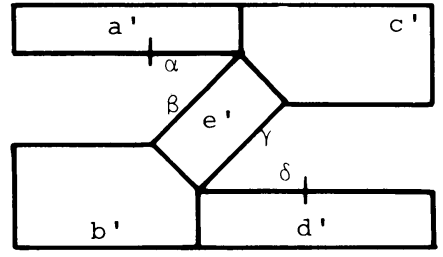

A neighborhood of $S^{\prime}$

(a)

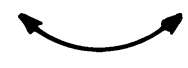

FIGURE 3.5

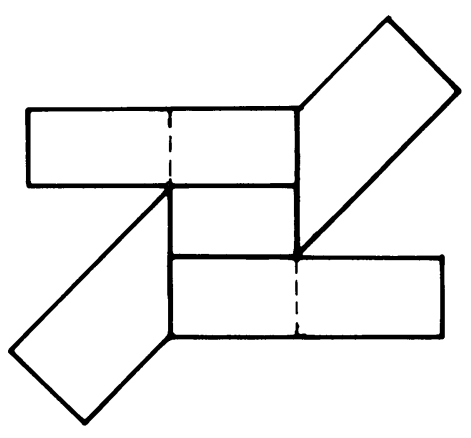

A neighborhood of $\mathrm{S}$

(b)

Now, return to the bigon track $T$ so that $w^{2}(T)$ collapses to $T$ and consider the bifoliated neighborhood $\left(N, \mathscr{F}, \mathscr{F}{ }^{\perp}\right)$. Apply the homeomorphism $w^{2}$ to this neighborhood to obtain a bifoliated neighborhood of $w^{2}(T)$ in $F$. We furthermore apply a "Grötsch map" to each rectangle expanding by a factor $\lambda$ in the horizontal and contracting by a factor $\lambda^{-1}$ in the vertical to obtain a bifoliated neighborhood $\left(M, \mathscr{G}, \mathscr{G}^{\perp}\right)$ of $w^{2}(T)$. It is clear that the sequence of collapses from $w^{2}(T)$ to $T$ determines a sequence of zippers on $\left(M, \mathscr{G}, \mathscr{G}^{\perp}\right)$, and the resulting bifoliated neighborhood is isotopic to $\left(N, \mathscr{F}, \mathscr{F}{ }^{\perp}\right)$. Indeed, consider the sequence of splits from $T$ to $w^{2}(T)$. Begin with the neighborhood $(N, \mathscr{F}, \mathscr{F} \perp)$ and perform the inverse of a zipper for each split. Since the inverse of a zipper affects metrics (and measures) by the appropriate linear maps, the "unzipped" neighborhood agrees with $\left(M, \lambda^{-1} \mathscr{G}, \lambda \mathscr{G}^{\perp}\right)$, as was asserted.

To define the map $f$ on $N$, let $w^{2}$ act on $N$ and then apply the Grötsch maps as before. Perform the zippings to again obtain $(N, \mathscr{F}, \mathscr{F} \perp)$. Extend $f$ to a map of $F$ in the natural way and remark that $\left.f\right|_{N}$ is one-to-one except over a locus contained in the singular leaves of $\mathscr{F}$; over this locus, $\left.f\right|_{N}$ is two-to-one. Finally, $f$ is homotopic to $w^{2}$ since these maps have exactly the same action on the bigon track $T$ up to homotopy, and $T$ fills $F$.

We next claim that $\left.f\right|_{N}$ has a unique periodic point in each smooth edge of $\partial N$. To see this, we note that $f$ is one-to-one over each smooth edge; indeed, if $E$ is such an edge, then $f(E)$ contains exactly one such smooth edge by construction. Therefore, $f$ induces a permutation of the smooth edges of $N$, so for a given edge $E$, there is some iterate $f^{k}$ of $f$ so that $E \subset f^{k}(E)$. By the expanding property $f(\mathscr{F})=\lambda \mathscr{F}$, it follows that there is a unique periodic point.

It follows that the foliation $\mathscr{F}$ on $N$ has no saddle-connections (i.e., leaves connecting singular points); for if there were a saddle-connection, then we might extend it to an arc contained in a leaf of $\mathscr{F}$ and connecting the periodic points of $f$ in adjacent edges of $\partial N$. This arc would be invariant under some iterate of $f$, contradicting the contracting property $f\left(\mathscr{F}^{\perp}\right)=\lambda^{-1} \mathscr{F} \perp$.

Thus, $f$ (and hence $w^{2}$ ) preserve an arational foliation with dilatation $\lambda>1$, and standard arguments in [FLP] then guarantee that $w^{2}$ is $\mathbf{p A}$. Rather than applying this reasoning, however, we prefer to explicitly construct a map $\psi$ homotopic to 
$w^{2}$ and transverse measured foliations $\mathscr{F}_{u}$ and $\mathscr{F}_{s}$ on $F$ with $\psi\left(\mathscr{F}_{u}\right)=\lambda \mathscr{F}_{u}$ and $\psi\left(\mathscr{F}_{s}\right)=\lambda^{-1} \mathscr{F}_{s}$. This approach requires a further technical claim as follows.

Suppose $E$ and $E^{\prime}$ are smooth edges of $\partial N$ which are incident on a common singular point $v$ of $N$, and let $\xi \in E$ and $\xi^{\prime} \in E^{\prime}$ denote the periodic points of $f$. (See Figure 3.6.) Let $D$ denote $\mathscr{F}^{\perp}$-distance along leaves of $\mathscr{F}$. We claim that $D(\xi, v)=D\left(\xi^{\prime}, v\right)$. To see this, let $v_{j}=f^{k_{j}}(v), j=1,2, \ldots$ and $k_{1}<k_{2}<\cdots$, be the forward images of $v$ which lie on the singular leaf of $\mathscr{F}$ issuing forth from $v$, and let $\eta_{j} \in E, \eta_{j}^{\prime} \in E^{\prime}$ denote points of $f^{-k_{j}} v$. By the expanding property of $f$, we have

$$
\lim _{j \rightarrow \infty} \eta_{j}=\xi, \quad \lim _{j \rightarrow \infty} \eta_{j}^{\prime}=\xi^{\prime} .
$$

Meanwhile, $D\left(\eta_{j}, v\right)=\lambda^{-k_{j}} D\left(v, v_{j}\right)=D\left(\eta_{j}^{\prime}, v\right)$, and the claim follows.

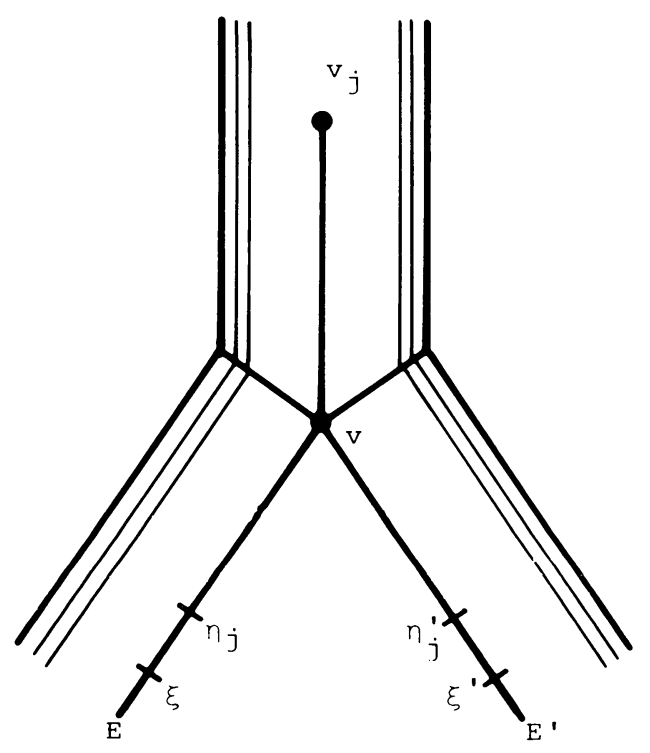

FIGURE 3.6

Finally, to obtain a homeomorphism of $F$ from the map $f$, we describe a quotient map $q$ : $N \rightarrow F$, which extends to a map on $F$ homotopic to $f$, so that $f \mid N$ takes fibers of $q$ to fibers of $q$; the map $q$ descends to the desired pA map. A fiber of $f$ is either a singleton or consists of a pair of points in adjacent smooth edges $E$ and $E^{\prime}$ of $\partial N$ whose $D$-distances to the common cusp $v$ of $E$ and $E^{\prime}$ agree. We thus $D$ isometrically identify the edges of each simply connected region $R$ complementary to $N$ while identifying each periodic point of $f$ in $\partial R$ to a single point; see Figure 3.7 .

The quotient surface is canonically homeomorphic to $F$ and inherits two transverse measured foliations $\mathscr{F}_{u}$ (from $\mathscr{F}$ ) and $\mathscr{F}_{s}$ (from $\mathscr{F}^{\perp}$ ). The action of $f$ on $N$ descends to a homeomorphism $\psi$ of the quotient surface with $\psi\left(\mathscr{F}_{u}\right)=\lambda \mathscr{F}_{u}$ and $\psi\left(\mathscr{F}_{s}\right)=\lambda^{-1} \mathscr{F}_{s}$. The theorem follows. 

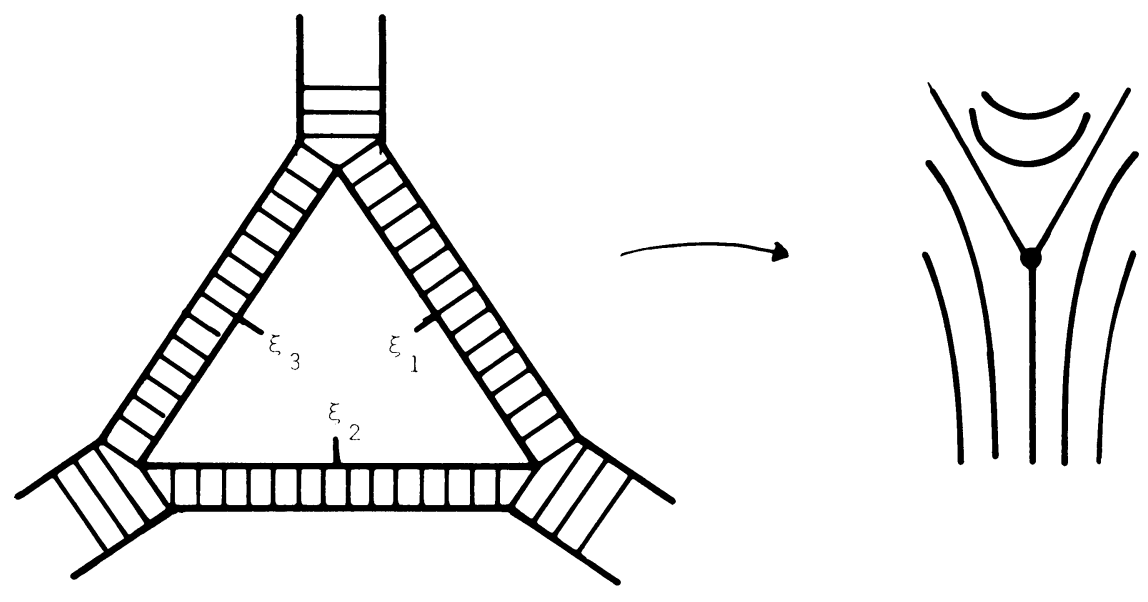

FIGURE 3.7

REMARKS. (1) The metric $\nu$ on $T$ does not determine a measured foliation on $F$ owing to the indeterminacy on complementary evengons. However, the action of $f$ on $N$ distinguishes fixed points in smooth edges of $\partial N$ and hence distinguishes $\mu^{*} \in$ $\mathscr{M}(\nu)$, which then determines $\mathscr{F}_{s}$. Further, in case there are only complementary oddgons for $T$, then we have seen that the (singleton) $\mathscr{M}(\nu)$ does in fact describe the foliation $\mathscr{F}_{s}$.

As a consequence of the proof of Theorem 3.1, we have the following criterion for recognizing $\mathrm{pA}$ maps.

COROLlaRY 3.2. Given a homeomorphism $\varphi$ of $F$, if there is a birecurrent bigon track $T \subset F$ filling $F$ so that $T$ splits to $\varphi(T)$ with Perron-Frobenius incidence matrix, then $\varphi$ is $p A$.

REMARK. The hypotheses of this corollary can probably be weakened to the setting that $T$ carries $\varphi(T)$ using ideas from [PP].

We recall

Theorem B (ThURSTON; SEE [T1 OR FLP, EXPOSE 13]). Suppose that $\mathscr{C}$ and $\mathscr{D}$ are singletons and satisfy the hypotheses of Theorem 3.1. One can give $F$ an affine structure with singularities so that the derivative of $\tau_{\mathscr{C}}^{ \pm 1}$ is $\left(\begin{array}{cc}1 & 0 \\ \pm 1 & 1\end{array}\right)$ and the derivative of $\tau_{\mathscr{D}}^{\mp 1}$ is $\left(\begin{array}{cc}1 & \pm r \\ 0 & 1\end{array}\right)$ in $\operatorname{PSL}(\mathbf{R})$, where $r \in \mathbf{R}^{+}$. If $w$ is any hyperbolic word in $\tau_{\mathscr{E}}$ and $\tau_{\mathscr{D}}$, then $w$ is $p A$.

Thus, our recipe is more general than the original construction of Thurston insofar as Theorem B requires twisting along all of the $\mathscr{C}$ curves, then all of the $\mathscr{D}$ curves, and so on; in contrast, our Theorem 3.1 allows twisting along only some of the $\mathscr{C}$ curves, then some of the $\mathscr{D}$ curves, and so on.

Now, let $S\left(\mathscr{C}^{+}, \mathscr{D}^{-}\right)$denote the semigroup with presentation

$$
S\left(\mathscr{C}^{+}, \mathscr{D}^{-}\right)=\left\langle e \in \mathscr{C} \cup \mathscr{D}: e \leftrightarrow e^{\prime} \text { if } e \cap e^{\prime}=\varnothing\right\rangle .
$$

$S\left(\mathscr{C}^{+}, \mathscr{D}^{-}\right)$is a quotient semigroup of $R\left(\mathscr{C}^{+}, \mathscr{D}^{-}\right)$, where the letter $e$ in $S\left(\mathscr{C}^{+}, \mathscr{D}^{-}\right)$corresponds to $\tau_{e}^{+1}$ if $e \in \mathscr{C}$ and to $\tau_{e}^{-1}$ if $e \in \mathscr{D}$. 
We conclude this section by describing a linear representation of $S\left(\mathscr{C}^{+}, \mathscr{D}^{-}\right)$. It will follow that the natural map $S\left(\mathscr{C}^{+}, \mathscr{D}^{-}\right) \rightarrow M C(F)$ is a monomorphism onto a semigroup of mapping classes (most of which are pA). For convenience, we restrict attention for the present to the setting where $\mathscr{C} \cup \mathscr{D}$ has no bad complementary regions (as in Figure 3.3) so that $T$ is a train track and no component of $\mathscr{C}$ or $\mathscr{D}$ is boundary- or puncture-parallel in $F$. With some simple modifications, part of our discussion applies also to the general case.

Let $\mathscr{E}=\left\{e_{k}\right\}_{1}^{n}=\mathscr{C} \cup \mathscr{D}$, and let $T$ denote the train track constructed in the proof of Theorem 3.1 with branches $\left\{b_{i}\right\}$. Each $e_{k}$ defines a curve $t\left(e_{k}\right) \subset T$ (as in the proof of Theorem 3.1) and a measure $\mu_{k}$ on $T$ defined by

$$
\mu_{k}\left(b_{i}\right)= \begin{cases}1, & b_{i} \subset t\left(e_{k}\right) \\ 0, & \text { otherwise }\end{cases}
$$

Let $H$ denote the convex hull of $\left\{\mu_{k}\right\}$ and the zero measure in the cone of measures on $T$. (In general, $H$ is a proper subcone of the cone of measures on T.) By definition, $\left\{\mu_{k}\right\}$ is a positive basis for $H$, and $H$ is clearly invariant under $S\left(\mathscr{C}^{+}, \mathscr{D}^{-}\right)$. Furthermore, the natural map $H \rightarrow \mathscr{M} \mathscr{T}_{0}(F)$ is an injection [HP], and we identify the set $H$ of measures on $T$ with the corresponding subset of $\mathscr{M} \mathscr{T}_{0}(F)$. Similarly, let $P H$ denote the projectivization of $H$, and identify $P H$ with the corresponding subset of $\mathscr{P} \mathscr{T}_{0}(F)$.

We describe the linear action of $S\left(\mathscr{C}^{+}, \mathscr{D}^{-}\right)$on $H$ in the basis $\left\{\mu_{k}\right\}$. Define the intersection matrix $A=\left(a_{i j}\right)$ by $a_{i j}=\operatorname{card}\left(e_{i} \cap e_{j}\right)$. Note that $A \geq 0$ and $A$ is symmetric. Define the matrix $A_{k}$ by

$$
\left(A_{k}\right)_{i j}= \begin{cases}A_{i j}, & i=k \\ 0, & \text { otherwise. }\end{cases}
$$

The action of $\tau_{e_{k}}^{\varepsilon}$ on $H$ with respect to the basis $\left\{\mu_{k}\right\}$ is given by $B_{k}=I+A_{k}$, where $\varepsilon=+1$ if $e_{k} \in \mathscr{C}$ and $\varepsilon=-1$ if $e_{k} \in \mathscr{D}$.

LEMMA 3.3. (a) Given $k, k^{\prime} \in\{1, \ldots, n\}$ so that $B_{k}^{-1} \mathbf{y}, B_{k^{\prime}}^{-1} \mathbf{y}>0$, then we have $a_{k k^{\prime}}=0$. Furthermore, for all $\mathbf{y}>0, B_{k}^{-1} B_{k^{\prime}}^{-1} \mathbf{y}>0$ provided $k \neq k^{\prime}$.

(b) If $M=\prod_{i=1}^{N} B_{k_{i}}$, then the $j$ th row of $M$ differs from the $j$ th unit basis vector if and only if $j \in\left\{k_{i}\right\}_{1}^{N}, j=1, \ldots, n$.

PROOF. (a) Note that $B_{k}^{-1}=I-A_{k}$. Thus, $\mathbf{y}=\left(y_{1}, \ldots, y_{n}\right)>A_{k} \mathbf{y}, A_{k^{\prime}} \mathbf{y}$; hence $y_{k}>a_{k k^{\prime}} y_{k^{\prime}}$ and $y_{k^{\prime}}>a_{k k^{\prime}} y_{k}$. This is absurd unless $a_{k k^{\prime}}=0$. For the second part, since $a_{k k^{\prime}}=0$, it follows that $B_{k}^{-1} B_{k^{\prime}}^{-1}=I-A_{k}-A_{k^{\prime}}$. Now $y_{k}>A_{k} \mathbf{y}$ and $y_{k^{\prime}}>A_{k^{\prime}} \mathbf{y}$ by hypothesis, so $\mathbf{y}>A_{k} \mathbf{y}+A_{k^{\prime}} \mathbf{y}$, as desired.

(b) Is immediate since each row of $A$ has a nonzero entry by hypothesis.

THEOREM 3.4. The action of $S\left(\mathscr{C}^{+}, \mathscr{D}^{-}\right)$on $H$ admits a faithful representation as a semigroup of invertible (over Z) positive (mostly Perron-Frobenius) matrices.

PROOF. We must check the faithfulness of our representation. Suppose, then, that $w, w^{\prime} \in S\left(\mathscr{C}^{+}, \mathscr{D}^{-}\right)$are represented by the products $B=\prod_{1}^{N} B_{k_{i}}=\prod_{1}^{N^{\prime}} B_{k_{i}^{\prime}}$, respectively, and let $\mathbf{y} \geq 0$ correspond to the unstable projective class of measured foliations invariant by the mapping class $[w]=\left[w^{\prime}\right]$. The proof that $w=w^{\prime}$ is by induction on $\max \left\{N, N^{\prime}\right\}$, and if $\max \left\{N, N^{\prime}\right\}=1$, then the result follows trivially. 
Suppose that $N \geq N^{\prime}$. By Lemma 3.3b, $k_{1} \in\left\{k_{i}^{\prime}\right\}_{1}^{N^{\prime}}$; moreover, if $I$ is the least index so that $k_{1}=k_{I^{\prime}}^{\prime}$ then it follows from Lemma 3.3a that $a_{k_{t}^{\prime} k^{\prime}}=0$ for all $k^{\prime}=k_{i}^{\prime}, i=1, \ldots, I$. Thus, we may commute the $I$ th letter of $w^{\prime}$ to the first, and the induction step follows on applying $B_{k_{1}}^{-1}$ to each of $\prod_{1}^{N} B_{k_{i}}$ and $\prod_{1}^{N^{\prime}} B_{k_{i}^{\prime}}$.

REMARK. In particular, if $\mathscr{C}=$ \{meridian $\}$ and $\mathscr{D}=\{$ longitude $\}$ on the punctured torus, then the determination of $w$ from $\mathbf{x}$ is well known to reduce to the computation of simple continued fractions. In analogy, Theorem 3.4 suggests the generalized continued fractions of Jacobi and Perron. (See, for example, [Be].)

COROLlaRY 3.5. The natural map $S\left(\mathscr{C}^{+}, \mathscr{D}^{-}\right) \rightarrow M C(F)$ is a monomorphism onto a semigroup of (mostly $p A)$ mapping classes.

A standard application of the Euclidean algorithm to $\mathrm{SL}_{2}(\mathbf{Z})$ shows that all pA mapping classes on the torus-minus-a-disc arise from our construction, and it is tempting to ask whether our recipe gives virtually all pA mapping classes. Precisely, if $\psi$ is pA, then $\psi^{n} \in S\left(\mathscr{C}^{+}, \mathscr{D}^{-}\right)$for some $n, \mathscr{C}$ and $\mathscr{D}$. A characterization of the pA maps we have constructed is difficult because of the complexity of the set of appropriate pairs $(\mathscr{C}, \mathscr{D})$ on $F$. The following example shows that our recipe does not construct all pA maps.

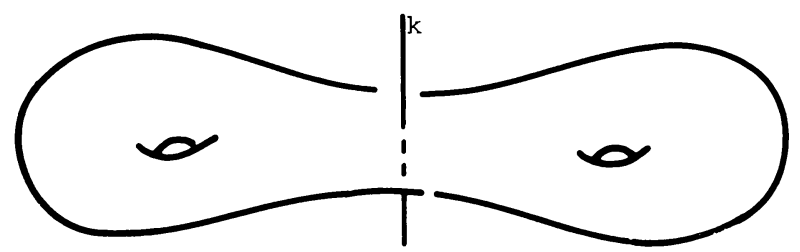

FIGURE 3.8

EXAmPLE. Consider the curves $\left\{c_{i}\right\}_{1}^{3}$ and $\left\{d_{i}\right\}_{1}^{3}$ on the two-holed torus $F_{2}$ indicated in Figure 3.1. Define a homeomorphism $\phi: F_{2} \rightarrow F_{2}$ by composing $\tau_{c_{3}} \circ \tau_{c_{I}} \circ \tau_{d_{1}}^{-1} \circ \tau_{d_{2}}^{-1} \circ \tau_{c_{2}}$ with rotation-by- $\pi$ about the line $k$ in Figure 3.8. Certainly $\phi^{2}$ arises from our construction, while $\phi$ does not. Indeed, all of the pA maps we have constructed fix the singular points of the invariant foliations $\mathscr{F}_{u}$ and $\mathscr{F}_{s}$ (and the separatrices based at each singular point), while $\phi$ above permutes them.

One approach to the conjecture is as follows. If $w$ is $\mathrm{pA}$, then let $l(w)$ denote the unstable projective class of measured foliations fixed by $w$. Denote by $D$ the union over all $\mathscr{C}$ and $\mathscr{D}$ of $\left\{l(w): w \in S\left(\mathscr{C}^{+}, \mathscr{D}^{-}\right)\right.$is pA $\}$. An equivalent formulation of the conjecture is that $D$ is the set of all unstable classes of tracks of pA maps. It is easy to show that $D$ is dense in $\mathscr{P} \mathscr{T}_{0}(F)$ using the fact [HP] that simple closed curves are dense in $\mathscr{P} \mathscr{T}_{0}(F)$. One is thus led to consider the closure $L\left(\mathscr{C}^{+}, \mathscr{D}^{-}\right)$ in $\mathscr{P} \mathscr{T}_{0}(F)$ of $\left\{l(w): w \in S\left(\mathscr{C}^{+}, \mathscr{D}^{-}\right)\right\}$. Each $L\left(\mathscr{C}^{+}, \mathscr{D}^{-}\right)$can be shown to be a closed, perfect, $S\left(\mathscr{C}^{+}, \mathscr{D}^{-}\right)$-invariant, nowhere dense "limit" set. Since $M C(F)$ acts ergodically on $\mathscr{P} \mathscr{T}_{0}(F)[\mathbf{M a}]$, it is unlikely that any $L\left(\mathscr{C}^{+}, \mathscr{D}^{-}\right)$has positive measure.

4. We extend our recipe for constructing pA maps into the setting of nonorientable surfaces. The proof, which we will omit, is essentially the same as before. We begin with some remarks on Dehn twists in nonorientable surfaces. 
Suppose that $c$ is a two-sided curve embedded in a (possibly) nonorientable surface $F$ so that $c \subset F$ has trivial normal bundle $\nu_{c}$. Let $\theta: \nu_{c} \rightarrow A=S^{1} \times[-1,1]$ be a trivialization of $\nu_{c}$ onto the standard oriented annulus $A$ with $c=S^{1} \times\{0\}$. We define the $\theta$-Dehn twist $\tau_{c}^{\theta}$ along $c$ to be the identity off of $\nu_{c}$, and on $\nu_{c}$ we define

$$
\tau_{c}^{\theta}(x)=\theta^{-1}\left(\left[p_{1} \circ \theta(x)\right] e^{\left\{\left[p_{2} \circ \theta(x)\right]+1\right\} i \pi}, p_{2} \circ \theta(x)\right),
$$

where $p_{j}$ denotes projection onto the $j$ th factor in $A, j=1,2$. Thus, if $F$ is oriented, $\tau_{c}^{\theta}=\tau_{c}^{+1}$ if $\theta$ is orientation-preserving, and $\tau_{c}^{\theta}=\tau_{c}^{-1}$ if $\theta$ is orientation-reversing.

Suppose that $c$ and $d$ are each two-sided embedded curves in $F$ with trivializations $\theta_{1}: \nu_{c} \rightarrow A$ and $\theta_{2}: \nu_{d} \rightarrow A$; suppose, moreover, that $p \in c \cap d$. We say $\theta_{1}$ and $\theta_{2}$ are inconsistent at $p$ if the pull-backs by $\theta_{1}$ and $\theta_{2}$ of the orientation on $A$ to a coordinate patch about $p$ disagree.

THEOREM 4.1. Suppose $\mathscr{E}=\left\{e_{i}\right\}^{n}$ is a collection of two-sided curves (no two of which are parallel) embedded in $F$ which intersect minimally and fill $F$. Suppose also that $\theta=\left\{\theta_{1}\right\}^{n}$ is a collection of trivializations of the normal bundles to $e_{i} \subset F$ that are inconsistent at each point of $e_{i} \cap e_{j}, i \neq j$. Let $R(\mathscr{E}, \theta)$ denote the semigroup generated by $\left\{\tau_{e_{i}}^{\theta_{i}}\right\}_{1}^{n}$. If $w \in R(\mathscr{E}, \theta)$, then all the component maps of (the isotopy class of) $w$ are $p A$, and (the isotopy class of) $w$ is itself $p A$ if each $\tau_{e_{i}}^{\theta_{i}}$ occurs at least once in $w$.

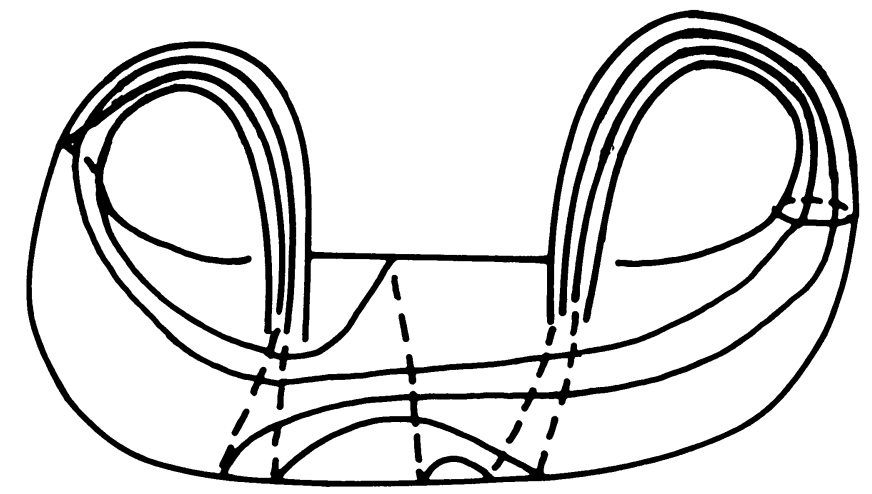

FIGURE 4.1

EXAMPLE. Let $\mathscr{E}$ denote the collection of three curves illustrated in Figure 4.1 on the connected sum of two Klein bottles. It is easy to find inconsistent trivializations and hence construct pA maps.

REMARKS. (1) One reason that a pA map on a nonorientable surface is interesting is that Bonahon introduced a notion of minimality of surface maps in his computation [Bo] of the cobordism group of diffeomorphisms of oriented surfaces. A nonminimal (in fact null-cobordant) diffeomorphism is constructed by extending one of the pA maps of the example to the twisted $I$-bundle over the connected sum of two Klein bottles and restricting to the boundary three-holed torus.

(2) On each of the projective plane, Klein bottle, and torus-sum-projective-plane there are unique simple curves with special properties. Using the characterization 
of pA maps given in the Introduction, it follows that the connected sum of two Klein bottles is the simplest nonorientable surface which supports a pA map.

(3) The nonorientable analogues of Theorem 3.4 and Corollary 3.5 are proved as before.

\section{REFERENCES}

[A] D. Anosov, Geodesic flows on compact Riemannian manifolds of negative curvature, Trudy Mat. Inst. Steklov 90 (1967)=Proc. Steklov Inst. Math. 90 (1969).

[AY] P. Arnoux and J. Yoccoz, Construction de diffeomorphisme pseudo-Anosov, C. R. Acad. Sci. Paris 292 (1981), 75-78.

[Be] L. Bernstein, The Jacobi-Perron algorithm-its theory and applications, Lecture Notes in Math., vol. 207, Springer-Verlag, 1971.

[Bo] F. Bonahon, Cobordism of automorphisms of surfaces, Ann. Sci. École Norm. Sup. (4) 16 (1983), 237-270.

[FLP] A. Fathi, F. Laudenbach, V. Poenaru et al., Travaux de Thurston sur les surfaces, Astérisque 30 (1979), 66-67.

[Ga] F. Gantmacher, Theory of matrices, Chelsea, 1959.

[Gi] J. Gilman, On the Nielsen type and the classification for the mapping class group, Adv. in Math. 40 (1981), 68-96.

[HP] J. Harer and R. Penner, Combinatorics of train tracks, preprint.

[HT] M. Handel and W. Thurston, New proofs of some results of Nielsen, Adv. in Math. 56 (1985), 173-191.

[K] S. Kerckhoff, The asymptotic geometry of Teichmuller space, Topology 19 (1980), 23-41.

[Ma] H. Masur, Interval exchange transformations and measured foliations, Ann. of Math. (2) 115 (1982), 169-200.

[Mi] R. Miller, Nielsen's viewpoint on geodesic laminations, Adv. in Math. 45 (1982), 189-212.

[N] J. Nielsen, Untersuchungen zur Topologie der geschlossenen zweiseitigen Flachen, Acta Math. I 50 (1927), 189-358; II 53 (1929), 1-76; and III 58 (1932), 87-167.

[Pa] A. Papadopoulos, Réseaux ferroviaires, diffeomorphismes pseudo-Anosov et automorphismes symplectique de l'homologie d'une surface, Publ. Math. Orsay 83-103, 1983.

[PP] A. Papadopoulos and R. C. Penner, A characterization of pseudo-Anosov foliations, Pacific J. Math. 130 (1987), 359-377.

[T1] W. Thurston, On the geometry and dynamics of diffeomorphisms of surfaces. I, preprint.

[T2] _ , The geometry and topology of three-manifolds, Princeton lecture notes, 1978.

[T3] — Lecture notes from Boulder, Colorado, 1981, taken by W. Goldman.

[T4] _ Three dimensional manifolds, Kleinian groups and hyperbolic geometry, Bull. Amer. Math. Soc. 6 (1982), 357--381.

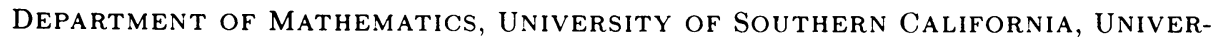
Sity PARK MC-1113, LOS ANGEles, CALIFORNia 90098-1113 